\title{
Randomization Documentation
}

National Cancer Institute

\section{Source}

National Cancer Institute. Randomization Documentation. NCI Thesaurus. Code

C115722.

Records pertaining to the randomization process of a clinical trial. 